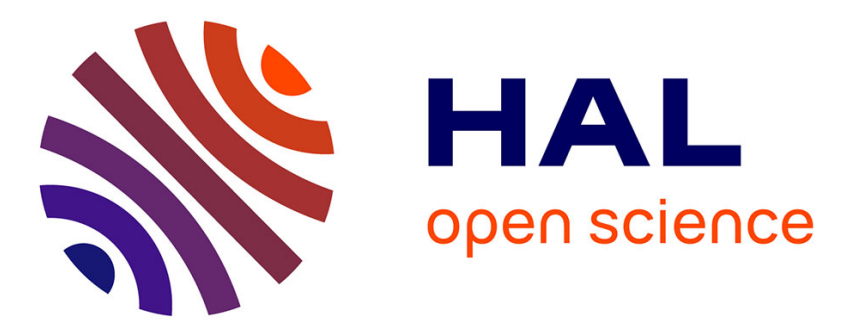

\title{
Scaling laws in axisymmetric magnetohydrodynamic duct flows
}

Alexandre Poyé, Olivier Agullo, Nicolas Plihon, Wouter J.T. Bos, Victor Désangles, Guillaume Bousselin

\section{To cite this version:}

Alexandre Poyé, Olivier Agullo, Nicolas Plihon, Wouter J.T. Bos, Victor Désangles, et al.. Scaling laws in axisymmetric magnetohydrodynamic duct flows. Physical Review Fluids, 2020, 5 (4), pp.043701. 10.1103/PhysRevFluids.5.043701 . hal-02167627v2

\section{HAL Id: hal-02167627 \\ https://hal.science/hal-02167627v2}

Submitted on 2 Apr 2020

HAL is a multi-disciplinary open access archive for the deposit and dissemination of scientific research documents, whether they are published or not. The documents may come from teaching and research institutions in France or abroad, or from public or private research centers.
L'archive ouverte pluridisciplinaire HAL, est destinée au dépôt et à la diffusion de documents scientifiques de niveau recherche, publiés ou non, émanant des établissements d'enseignement et de recherche français ou étrangers, des laboratoires publics ou privés. 


\title{
Scaling laws in axisymmetric magnetohydrodynamic duct flows
}

\author{
A. Poyé $\odot,{ }^{1,2}$ O. Agullo $\odot,{ }^{2}$ N. Plihon $\odot,{ }^{1}$ W. J. T. Bos $\odot,{ }^{3}$ V. Desangles,${ }^{1}$ and G. Bousselin ${ }^{1}$ \\ ${ }^{1}$ Univ Lyon, ENS de Lyon, Univ Claude Bernard Lyon 1, CNRS, Laboratoire de Physique, \\ F-69342 Lyon, France \\ ${ }^{2}$ PIIM, UMR No. 7345, CNRS, Aix-Marseille Université, 13397 Marseille, France \\ ${ }^{3}$ LMFA, CNRS, Ecole Centrale de Lyon, Université de Lyon, 69130 Écully, France
}

(Received 8 May 2019; accepted 14 February 2020; published 1 April 2020)

\begin{abstract}
We report on a numerical study of axisymmetric flow of liquid metal in a circular duct with a rectangular cross section. The flow is forced through the combination of an axial magnetic field and a radial current. Sweeping a wide range of forcing parameters, we identify the different regimes which characterize the flows and explicate the associate scaling laws. Results from different studies in the literature are interpreted in the light of our numerical simulations.
\end{abstract}

DOI: 10.1103/PhysRevFluids.5.043701

\section{INTRODUCTION}

The magnetohydrodynamic (MHD) flow through a toroidal duct is one of the elementary academic flows to study the behavior of liquid metals. It proves experimentally convenient since it is limited in size and of relatively low complexity, numerically convenient since no inlet-outlet conditions have to be specified, and analytically convenient due to its symmetry. Electrically driven flows have been proposed for magnetorotational instability (MRI) experiments [1,2], MRI being an essential ingredient in the dynamics of accretion disks. Duct MHD flows give rise to a number of competing mechanisms due to the simultaneous presence of rotation, magnetic field, and possibly turbulence. Its description, even in the laminar case, is far more involved than simple duct flow of a nonconducting fluid. A number of different instabilities can be triggered and the mean flows are not even well identified.

The possibility to observe MRI in electrically driven annular duct flow and Taylor-Couette flow has been investigated both experimentally and numerically [2-4]. Further investigations of electrically driven duct flow have been reported in the literature [5-11]. One important observation in these works is that a large number of regimes can be observed, depending on the strength of the magnetic field, electric current, fluid properties, and geometry of the duct.

Early studies [1] focused on the diffusive regime, which is most easily treated analytically. The more complex case where inertial effects become important was addressed more recently [2,12], both numerically and analytically. In a recent attempt [13] to sort out the different possible flows, experiments were carried out to distinguish between inertial and diffusive regimes, focusing on the influence of the interaction parameter and flow geometry. In the latter experiment, as in most other experimental devices, the Hartmann number Ha, which compares the Lorentz force to the viscous effects, lies in the range [400, 4000] [1,10].

In the experiments, reaching regimes in which inertia plays the dominant role requires strong magnetic fields (typically of the order from 1 to $10 \mathrm{~T}$ ) over a few centimeters, which remains a technical challenge, not attained in most studies, an exception being the reference work of Moresco and Alboussière [8]. The limit of low Hartmann number $\mathrm{Ha} \ll 1$ has been investigated only with electrolytes and small current [14,15].

In this study we aim to identify and characterize the flow regimes of electrically driven duct flows, for given fluid properties. For this purpose, we will carry out simulations over a wide range 


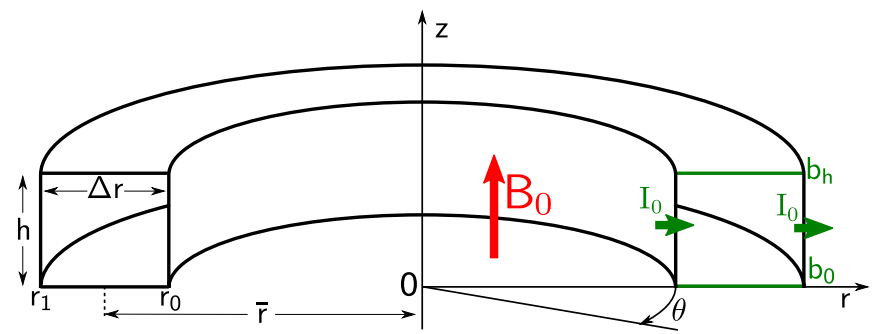

FIG. 1. Scheme of the annular duct. The axisymmetric 2D simulations presented in this study are displayed on the $(r, z)$ plane.

of Hartmann numbers (high or low magnetic field) and flow drive (high or low electrical drive), focusing on the specific case of axisymmetric flow patterns. More specifically, we characterize the various mean flows and analyze their features as a function of the main control parameters using scaling laws. We also present a study of the influence of the duct's geometry (aspect ratio of the cross section and mean radius of the duct) on the nature of the flows. Finally, we focus on a comparison with experimental results. All results presented in this article have been obtained assuming that the flow remains axisymmetric before the transition to turbulence. This assumption allows us to explore the influence of the control parameters on the evolution of the flow pattern, spanning a wide range of values for the control parameters while keeping the computation time within affordable limits. However, it is well known that axisymmetry may be broken in nonturbulent regimes: A variety of nonaxisymmetric patterns are observed, for instance, in pure hydrodynamic Taylor-Couette flows [16,17] or MHD Taylor-Couette flows [18]. The scaling laws reported in the present study for axisymmetric regimes should thus be interpreted as indicative results; the possible emergence of axisymmetry breaking and its influence on the reported scaling laws would require full three-dimensional (3D) computations, well beyond the scope of this study.

In the next section we will describe the model and methods used. In Sec. III we will discuss the nature of mean turbulent flows and compare it with theoretical steady-state predictions. We discuss also the link between Dean vortices and boundary layers. In Sec. IV we characterize the different flow regimes and explicate the mean flow power laws. We also point out the influence of the geometry on the nature of the flows. In Sec. V we provide a comparison between experimental and numerical results. We summarize in Sec. VI.

\section{SETUP, METHODS, AND PARAMETERS}

\section{A. Geometry and governing equations}

We consider an annular channel of height $h$, inner radius $r_{0}$, and outer radius $r_{1}$, filled with an electrically conducting fluid and in which a flow is generated [mainly in the azimuthal $(\theta)$ direction] from the interaction of an imposed axial magnetic field of intensity $B_{0}$ and an electric current $I_{0}$ in the radial direction, as sketched in Fig. 1. The channel width will be refereed to as $\Delta r$ and the mean radius as $\bar{r}$. We will restrict our study to cases where the electric current is injected from the inner radial channel wall. The system is governed by the incompressible MHD equations

$$
\begin{gathered}
\rho \partial_{t} \mathbf{V}=-\rho \mathbf{V} \cdot \nabla \mathbf{V}-\nabla P+\mathbf{j} \times \mathbf{B}+\rho \nu \Delta \mathbf{V}, \\
\partial_{t} \mathbf{B}=\nabla \times(\mathbf{V} \times \mathbf{B})+\frac{\eta}{\mu_{0}} \Delta \mathbf{B}, \\
\nabla \cdot \mathbf{V}=\mathbf{0}, \\
\nabla \cdot \mathbf{B}=\mathbf{0},
\end{gathered}
$$


where $\mathbf{V}$ is the fluid velocity, $\mathbf{B}$ the magnetic field, $\mathbf{j}=\mu_{0}^{-1} \nabla \times \mathbf{B}$ the current density, $\mu_{0}$ the magnetic permeability of vacuum, $\rho$ the fluid density, $\eta$ the fluid resistivity, and $v$ the fluid kinematic viscosity. The magnetic and velocity fields are normalized by the applied axial magnetic field $B_{0}$ and by the Alfvén speed $V_{A}=B_{0} / \sqrt{\rho \mu_{0}}$, respectively, while spatial distances are normalized by the channel height $h$. Five dimensionless numbers govern the dynamics of the system, namely, the magnetic Prandtl number $\mathrm{Pm}=\mu_{0} v / \eta$ (which compares the kinematic diffusivity to the magnetic diffusivity), the geometrical aspect ratios $\bar{r} / h$ and $\epsilon^{-1}=\Delta r / h$, the Hartmann number $\mathrm{Ha}=$ $h B_{0} / \sqrt{\rho \eta v}$ (where $\mathrm{Ha}^{2}$ compares the Lorentz force to the viscous dissipation), and a normalized current $I_{N}=\mu_{0} I_{0} / \sqrt{\rho \eta \nu}$. We will restrict our study to liquid mercury at room temperature, which means a constant and very low value of Pm. The results presented in the rest of this article will focus on the influence of the Hartmann number and the normalized current on the features of the electromagnetically driven flow. The influence of the geometry on the flow is also investigated through the aspect ratios.

\section{B. Axisymmetric formulation, boundary conditions, and numerical method}

Assuming axisymmetry [19] and following the problem formulation derived earlier by Khalzov et al. [2], the velocity and magnetic fields are expressed using stream functions as

$$
\begin{gathered}
\mathbf{V} / V_{A}=\frac{u(r, z)}{r} \mathbf{e}_{\theta}+\frac{1}{r} \nabla w(r, z) \times \mathbf{e}_{\theta}, \\
\mathbf{B} / B_{0}=\mathbf{e}_{\mathbf{z}}+\sqrt{\operatorname{Pm}}\left(\frac{b(r, z)}{r} \mathbf{e}_{\theta}+\frac{1}{r} \nabla \psi(r, z) \times \mathbf{e}_{\theta}\right),
\end{gathered}
$$

where $r$ and $z$ are the radial and axial coordinates, respectively, $u$ is the toroidal angular momentum of the flow, $w$ is the velocity stream function, $b$ is the angular toroidal magnetic field, and $\psi$ is the toroidal potential vector component. The right-hand-side quantities in Eqs. (5) and (6) are dimensionless.

Equations (1)-(4) are then recast as

$$
\begin{aligned}
& \partial_{t} u=\Delta^{*} u+\mathrm{Ha}_{z} b+\frac{\mathrm{Ha}}{r \sqrt{\mathrm{Pm}}}\{u, w\}-\frac{\mathrm{Ha} \sqrt{\mathrm{Pm}}}{r}\{b, \psi\}, \\
& \partial_{t} b=\triangle^{*} b+\mathrm{Ha}_{z} u+r \operatorname{Ha} \sqrt{\operatorname{Pm}}\left\{\psi, \frac{u}{r^{2}}\right\}-r \operatorname{Ha} \sqrt{\mathrm{Pm}}\left\{w, \frac{b}{r^{2}}\right\}, \\
& \partial_{t} \triangle^{*} w=\Delta^{*} \triangle^{*} w+\mathrm{Ha} \partial_{z} \Delta^{*} \psi-\frac{\mathrm{Ha}}{\sqrt{\mathrm{Pm}}}\left(r\left\{w, \frac{\triangle^{*} w}{r^{2}}\right\}+\frac{1}{r^{2}} \partial_{z} u^{2}\right) \\
& -\mathrm{Ha} \sqrt{\mathrm{Pm}}\left(r\left\{\psi, \frac{\triangle^{*} \psi}{r^{2}}\right\}+\frac{1}{r^{2}} \partial_{z} b^{2}\right), \\
& \partial_{t} \psi=\Delta^{*} \psi+\mathrm{Ha} \partial_{z} w+\frac{\mathrm{Ha} \sqrt{\mathrm{Pm}}}{r}\{\psi, w\},
\end{aligned}
$$

where $\Delta^{*} w$ is the toroidal vorticity, $\Delta^{*}$ is the Laplacian in cylindrical coordinates, and $\{\cdot, \cdot\}$ denotes the Poisson bracket

$$
\begin{gathered}
\triangle^{*} u=\partial_{z}^{2} u+\partial_{r}^{2} u-\frac{1}{r} \partial_{r} u, \\
\{u, w\}=\partial_{r} u \partial_{z} w-\partial_{r} w \partial_{z} u .
\end{gathered}
$$

The normalized injected current is then controlled in the simulations by the boundary conditions for the magnetic field $\mathbf{B}$ at the top and bottom walls of the channel. Indeed, the normalized 
current density

$$
\mathbf{j}(r, z)=\frac{\sqrt{\mathrm{Pm}}}{r}\left[-\partial_{z} b(r, z) \mathbf{e}_{\mathbf{r}}-\Delta^{*} \psi(r, z) \mathbf{e}_{\theta}+\partial_{r} b(r, z) \mathbf{e}_{\mathbf{z}}\right]
$$

is tangential to the top and bottom insulating walls and is perpendicular to side conductive walls. It implies the following boundary conditions on $b$ and $\psi$ :

$$
\begin{gathered}
\left.b(r)\right|_{z=0, h}=b_{0}, b_{h}, \\
\left.\partial_{z} b(z)\right|_{r_{0}, r_{1}}=0,0, \\
\left.\psi(r)\right|_{z=0, h}=\left.\psi(z)\right|_{r=r_{0}, r_{1}}=0, \\
\left.\triangle^{*} \psi(z)\right|_{r=r_{0}, r_{1}}=0, \\
\left.\partial_{r} \Delta^{*} \psi(r)\right|_{z=0, h}=0 .
\end{gathered}
$$

Here $b_{h}$ and $b_{0}$ are the values of $b$ at the top and the bottom of the duct, respectively. They fix the value of the injected current $I_{0}$ (in amperes) as

$$
I_{0}=\sqrt{\mathrm{Pm}} \frac{2 \pi}{\mu_{0}}\left(b_{0}-b_{h}\right) .
$$

Let us emphasize that those boundary conditions impose in fact that the total radial current flowing in the duct through any surface $r=$ const is constant and equal to $I_{0}$. No-slip boundary conditions for the velocity field at the duct walls impose

$$
\begin{gathered}
\left.u(z)\right|_{r=r_{0}, r_{1}}=\left.u(r)\right|_{z=0, h}=0, \\
\left.w(z)\right|_{r=r_{0}, r_{1}}=\left.w(r)\right|_{z=0, h}=0, \\
\left.\partial_{z} w(z)\right|_{r=r_{0}, r_{1}}=0, \\
\left.\partial_{r} w(r)\right|_{z=0, h}=0 .
\end{gathered}
$$

One salient feature of MHD flows is the presence of thin viscoresistive layers at the wall which imposes strong constraints on the discretization method. Indeed, Hartmann layers develop in the presence of a magnetic field perpendicular to solid boundaries (e.g., the top and bottom boundaries in Fig. 1) and have a normalized thickness of order $\mathrm{Ha}^{-1}$. Shercliff layers, on the other hand, develop in the presence of a magnetic field parallel to solid boundaries (e.g., the sidewalls in Fig. 1) and have a normalized thickness of order $\mathrm{Ha}^{-1 / 2}$. Both of these layers are vanishingly small at large Hartmann numbers.

The system is integrated using an explicit fourth-order Runge-Kutta temporal scheme combined with an inhomogeneous finite-difference scheme [20]. The inversion of the Laplacians is handled by a successive overrelaxation (SOR) algorithm [21]. The code is parallelized using the Message Passing Interface library. In order to properly resolve the Hartmann and Shercliff layers, the spatial mesh is refined close to the duct walls as

$$
\begin{gathered}
\delta r(r)=1-\exp \left[K_{r}\left(r_{0}-r\right)\right]-\exp \left[K_{r}\left(r-r_{1}\right)\right]+\exp \left[K_{r}\left(r_{0}-r_{1}\right)\right], \\
\delta z(z)=1-\exp \left(-K_{z} z\right)-\exp \left[K_{z}(z-h)\right]+\exp \left(-K_{z} h\right),
\end{gathered}
$$

where $\delta r$ and $\delta z$ are the radial and axial mesh sizes, respectively. The parameters $K_{r}$ and $K_{z}$ are adapted according to the device size and the value of Ha. The mesh refinement can exceed a factor of 10 at the boundaries for large Ha values. In total, we have carried out a few hundred simulations 
TABLE I. Values of nondimensional numbers within the range of parameters explored.

\begin{tabular}{lccc}
\hline \hline & $\begin{array}{c}\text { Hartmann } \\
\text { number } \\
h B_{0} / \sqrt{\rho \nu}\end{array}$ & $\begin{array}{c}\text { Reynolds } \\
\text { number } \\
h V_{\theta} / v\end{array}$ & $\begin{array}{c}\text { Inertial criterion } \\
\left(2 h^{2} / \bar{r}^{2}\right) \mathrm{Re} / \mathrm{Ha}^{2}\end{array}$ \\
Reference case & 25.9 & {$\left[254-\left(1.0 \times 10^{4}\right)\right]$} & {$[0.04-1.5]$} \\
$I_{0} \in[0.1-20] \mathrm{A}, I_{N} \in[0.003-0.65]$ & {$[0.26-258.6]$} & {$[35-1766]$} & {$\left[51.8-\left(2.6 \times 10^{-3}\right)\right]$} \\
$B_{0} \in[0.001-1] \mathrm{T}$ & 25.9 & {$[2870-13]$} & {$\left[8.4-\left(1.9 \times 10^{-7}\right)\right]$} \\
$\bar{r} \in[1-450] \mathrm{cm}$ & {$[6.5-827.7]$} & {$[285-6484]$} & {$[0.04-0.95]$} \\
$\Delta r=1 \mathrm{~cm}, h \in[0.25-32] \mathrm{cm}$ &
\end{tabular}

with typical $200 \times 200$ grid points. Time integration was carried out until statistically steady states were observed for a long enough time interval to converge the statistics.

\section{Numerical setup: Experimental control parameters or dimensionless numbers?}

The goal of the present article is to determine the amplitude of the azimuthal velocity as a function of the control parameters of the system. As emphasized above, the dynamics of the system is governed by five dimensionless numbers. However, focus is put on the influence of the Hartmann number (or equivalently the amplitude of the imposed magnetic field $B_{0}$ ) and the normalized current (or equivalently the imposed current $I_{0}$ at the sidewalls). Characterization of the flows can be presented as a function of either dimensionless numbers or experimental control parameters. Despite the elegance of a parameter scan as a function of dimensionless parameters, we have chosen to present our results as a function of the experimental control parameters encountered in experiments to allow direct comparison. Our approach is therefore to consider the material properties (density, electrical resistivity, and viscosity) of liquid mercury at room temperature and to study the influence of the values of the magnetic field, injected current, and the geometry of the experiment (aspect ratio and radius). The parametric values in the simulations are selected in order to assess and extend previous numerical and experimental studies [2,7,13].

In our parameter scans, unless explicitly mentioned, we will consider one particular reference case with experimentally convenient parameters and we will vary one control parameter at a time with respect to this case. This reference case is set with the following parameters: $B_{0}=0.1 \mathrm{~T}$, $h=1 \mathrm{~cm}, r_{0}=4 \mathrm{~cm}$, and $r_{1}=5 \mathrm{~cm}$. The injected current is $I_{0}=0.5$ A with $b_{h}=-b_{0}=0.13$. The fluid is mercury $\left(\rho=13550 \mathrm{~kg} / \mathrm{m}^{3}, v=1.14 \times 10^{-7} \mathrm{~m}^{2} / \mathrm{s}, \eta=9.68 \times 10^{-7} \Omega \mathrm{m}\right)$. In our simulations the magnetic field $B$ ranges from $10^{-3}$ to $1 \mathrm{~T}$, the current $I_{0}$ from 0.1 to $20 \mathrm{~A}$, the height $h$ from 0.25 to $32 \mathrm{~cm}$, and the radius $\bar{r}$ from 1 to $450 \mathrm{~cm}$.

In order to facilitate the comparison of our results with respect to other studies $[2,7,13]$, we present in Table I the parameter ranges that we have explored in terms of the Hartmann and Reynolds numbers and inertial criterion (see definition in Table I). Note that for our reference case $\mathrm{Ha}=25.9$, $\mathrm{Re}=1158, \mathrm{Pm}=1.48 \times 10^{-7}$, the Stuart number $N=\mathrm{Ha}^{2} / \mathrm{Re}=1.73$, and the inertial criterion $B_{a} N^{-1}=0.17$, where $B_{a}=2 h^{2} / \bar{r}^{2}$ is a geometric factor.

\section{ASYMPTOTIC STEADY-STATE FLOWS: BASIC PROPERTIES AND MECHANISMS}

Due to the substantial number of control parameters, no comprehensive understanding and consensus exists on the different flow regimes observed in electromagnetically driven annular duct flow. Before exploring the different possible regimes, we will study the low-magnetic-Prandtlnumber case addressed analytically and numerically in a previous study [2] in order to benchmark our computations. 

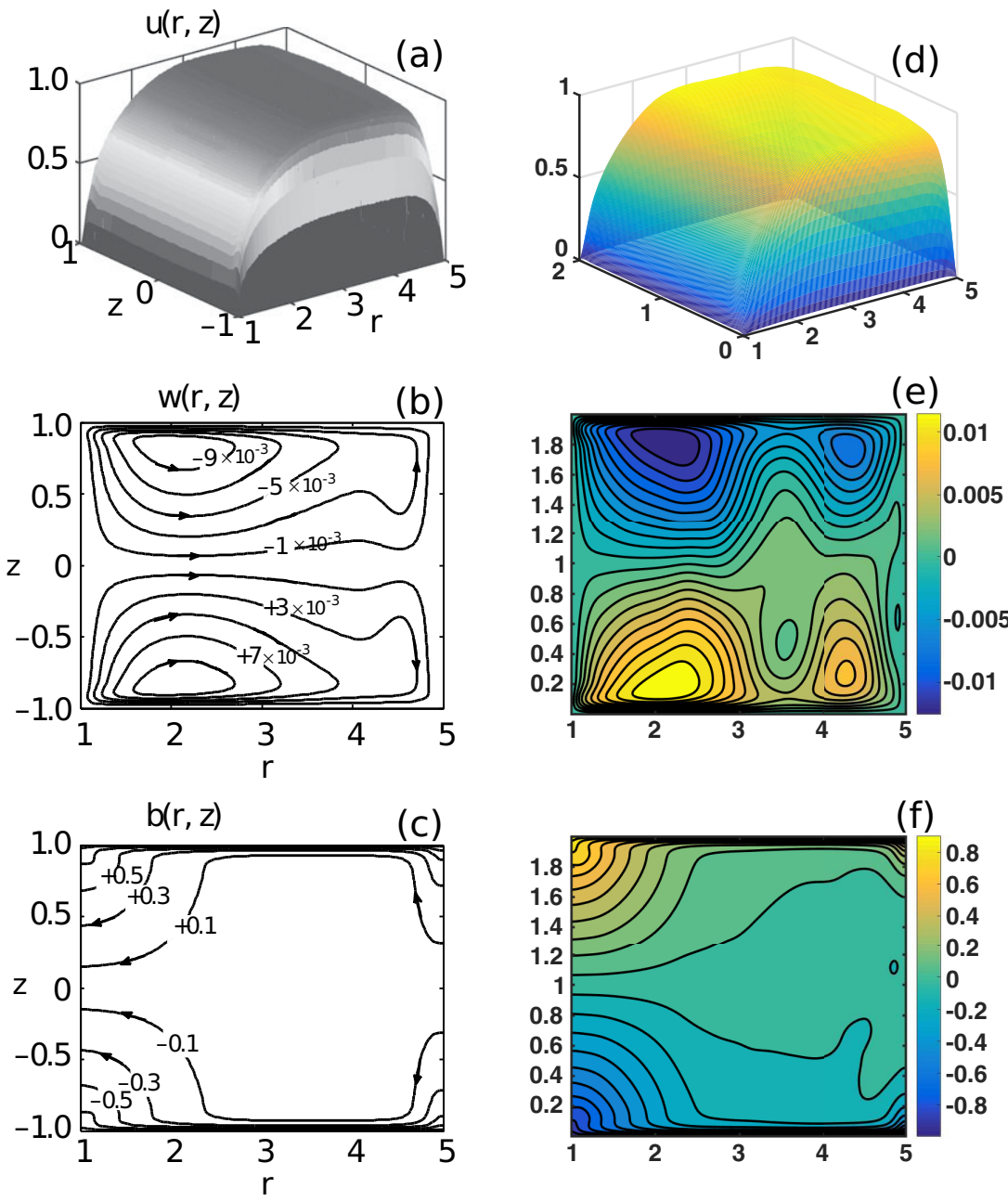

FIG. 2. Fields $u(r, z), w(r, z)$, and $b(r, z)$ for $h=2, r_{0}=1, r_{1}=5, \mathrm{Pm}=5.6 \times 10^{-5}$, and $\mathrm{Ha}=30$. (a)(c) Equilibrium solution of the model (7)-(10) without terms proportional to $\sqrt{\mathrm{Pm}}$. (d)-(f) Temporal average of the fields during the asymptotic state for the model (7)-(10).

\section{A. Low-magnetic-Prandtl-number limit: Existence of steady-state flows}

Analytical equilibrium solutions for the toroidal velocity component $u(r, z)$ were obtained in the quasistatic regimes [2] where the terms proportional to $\sqrt{\mathrm{Pm}}$ and temporal derivatives were neglected in Eqs. (7)-(10). In this limit, $\psi$ behaves as a passive scalar, the solution of $\triangle^{*} \psi+\mathrm{Ha}_{z} w=0$. The fields $w(r, z), b(r, z)$, and $\psi(r, z)$ may then be computed numerically. We present here a direct comparison with their results, for the equivalent parameters $\mathrm{Pm}=5.6 \times 10^{-5}$ and $\mathrm{Ha}=30$. First, we perform a successful benchmark using a global SOR method in our code, which computes the same time-independent solution reported in [2]. Then Fig. 2 compares this time-independent reference case [2] and the time-averaged steady-state fields obtained using our computations including the terms proportional to $\sqrt{\mathrm{Pm}}$. Both of the results shows similar features. We note the presence of very narrow Hartmann layers in the vicinity of and all along the horizontal walls, while the Shercliff layers at the vertical walls are less pronounced, as expected.

In the remainder of this study we will keep the fully-time-dependent solution with all the terms in Eqs. (7)-(10). As expected, temporal fluctuations develop around a statistically steady state. In 


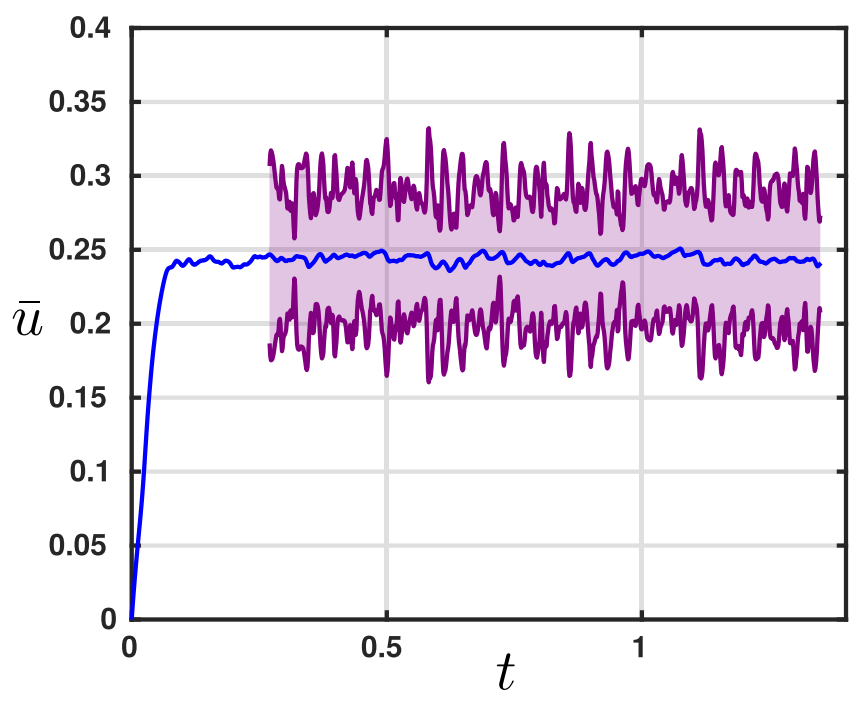

FIG. 3. Time evolution of $\bar{u}$ the spatial average of $u$, with the model (7)-(10). Surrounding purple curves are the spatial average of the standard deviation $\delta u$. The parameters are $\mathrm{Pm}=5.6 \times 10^{-5}, h=2, r_{0}=1, r_{1}=5$, and $\mathrm{Ha}=30$.

Fig. 3 we observe that the amplitude of the fluctuations of the mean toroidal flow $\delta u$ (purple zone) constitute approximately $40 \%$ the value of the mean flow $\bar{u}$. However, the time-averaged statistical steady state obtained in our simulations is equal to the quasistatic solution [2].

The study of the underlying instabilities and of the turbulent fluctuations is left for future work. However, this result shows that before those devices can be used to study the MRI, one needs to carefully assess the flow properties and in particular the other types of instabilities that might be present in the system. Let us emphasize, however, that the temporal fluctuations do not strongly modify the base flow and the time-averaged turbulent state is very similar to the previously described stationary flow [2].

In the remainder of this article we will investigate the time-averaged flow during the statistically steady state. We chose to keep the full model time dependent since it allows for a quantification of the fluctuating fields (left for future work). Unless other specified, all quantities denote in the following time averages. Volume averages will be specified, where needed, by angular brackets.

\section{B. Mean flow and origin of the boundary layers}

Let us consider the mean flow of the reference case introduced in Sec. II. Note that the flow is unsteady but develops weak fluctuations (less than $2 \%$ ) for this reference case. The time-averaged flow and magnetic potential are shown in Fig. 4. Clearly the boundary layers are wider than in the previous case, despite a similar Hartmann number. The poloidal magnetic field is much lower, as expected when the magnetic Prandtl number decreases. The amplitude of the poloidal flow $u$ is similar in both cases. The features reported in Fig. 4 allow us to identify the main mechanisms generating the flow, the current, and the Hartmann layer. The toroidal velocity $V_{\theta}=u / r$ is generated by the Lorentz force $j_{r} \times B_{0}$ along the $\theta$ direction. This dominant flow induces a poloidal magnetic field [the term $\mathrm{Ha}_{z} u$ in Eq. (8)] and creates vertical and radial currents from Eq. (13). The isovalue curves of the toroidal magnetic field $b$, displayed in Fig. 4(b), are also electrical current streamlines $\mathbf{j} \times \mathbf{d} \mathbf{M}=0(\psi \ll b)$. A fraction of current is expelled toward the insulating horizontal wall, i.e., the Hartmann layers. The vorticity $\omega=\nabla \times \mathbf{V} / V_{A} \approx r^{-1} \nabla u \times \mathbf{e}_{\theta}(w \ll u)$ is advected by the toroidal velocity $u$ and thus towards the horizontal wall, as seen in Fig. 4(a), the isocontours of $u$ being streamlines of the vorticity. The toroidal vorticity (and thus the poloidal component of the flow 

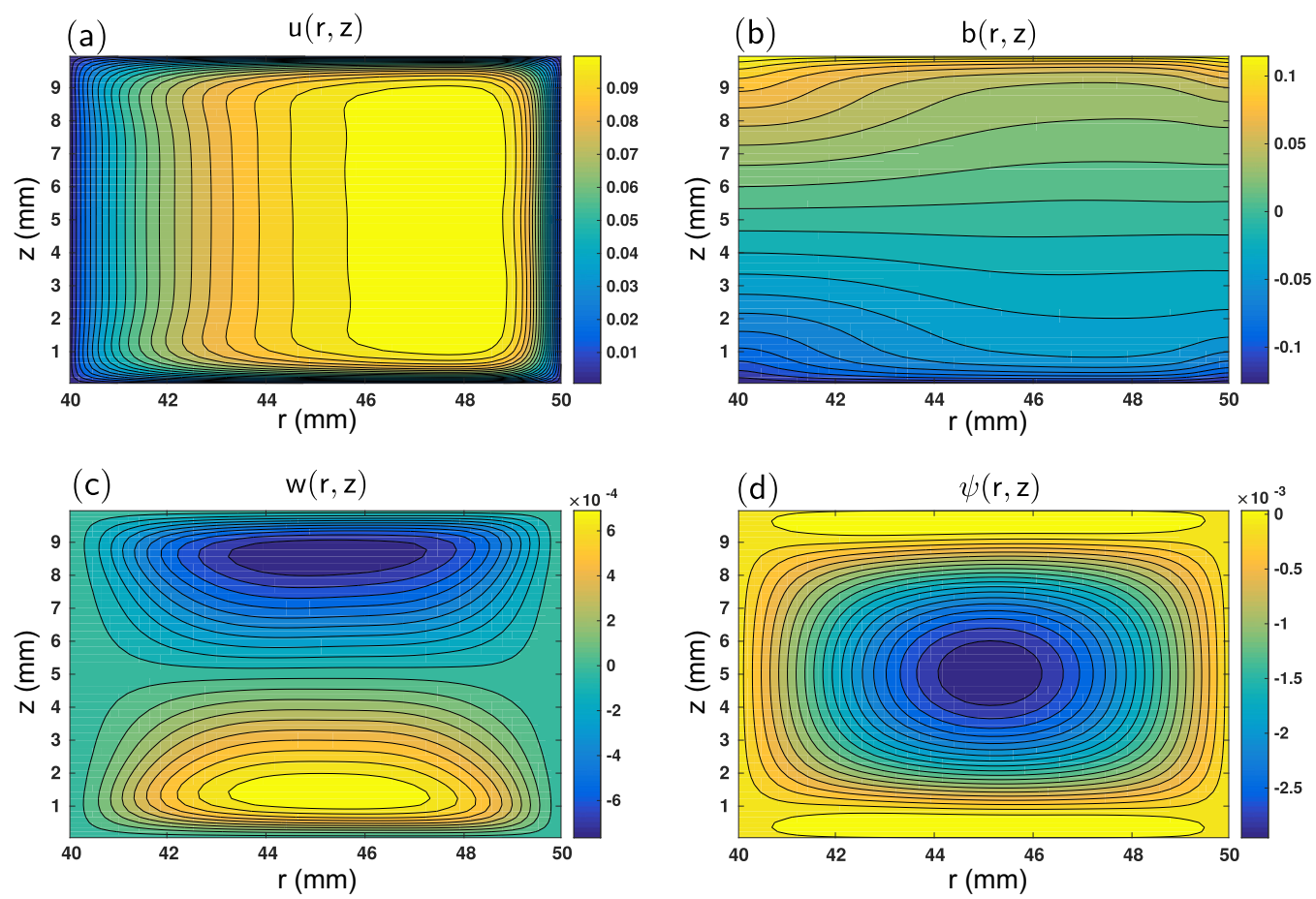

FIG. 4. Plot of the $(r, z)$ cross section of the flow and the current (a) $u(r, z)$, (b) $b(r, z),(\mathrm{c}) w(r, z)$, and (d) $\psi(r, z)$ for the reference case $\mathrm{Pm}=1.48 \times 10^{-7}$ and $\mathrm{Ha}=25.9$. The parameters are $B_{0}=0.1 \mathrm{~T}, I_{0}=$ $0.5 \mathrm{~A}, h=1 \mathrm{~cm}, r_{0}=4 \mathrm{~cm}$, and $r_{1}=5 \mathrm{~cm}$. The fluid is mercury $\left[\rho=13550 \mathrm{~kg} / \mathrm{m}^{3}, v=1.14 \times 10^{-7} \mathrm{~m}^{2} / \mathrm{s}\right.$, and $\left.\eta=\mu_{0}\left(7.7 \times 10^{-1}\right) \Omega \mathrm{m}\right]$.

$\left.r^{-1} \nabla w \times \mathbf{e}_{\theta}\right)$ is then generated from the centrifugal force $\left[r^{-2} \frac{H a}{\sqrt{P m}} \partial_{z} u^{2}\right.$ term in Eq. (9)] at the layer where $\partial_{z} \gg \partial_{r}$, as shown in Fig. 4(c). Isolines of $w$, up to the factor $r^{-1}$, correspond to streamlines of the poloidal flow $\mathbf{V}_{\perp \theta}$. Large convective rolls are observed on both sides of the horizontal midplane. The latter are generically referred to as Dean rolls in the literature [22-24] and play a dominant role in the generation of the Shercliff layers at the conducting walls. Note that the radial asymmetry of the flow $u(r, z)$ is linked to the Dean vortices which advect and then compress the flow towards the outer wall, and consequently amplify the outer Shercliff layer.

\section{WEAK AND MODERATE MAGNETIC-MACH-NUMBER FLOW REGIMES}

The features of the time-averaged MHD annular duct flow are usually analyzed from the balance of the dominant terms of Eq. (1). In previous works [1,2] it was emphasized that the inertialess regime is reached when

$$
2 R_{e} \ll \operatorname{Ha}^{2}(\bar{r} / h)^{2},
$$

and this defines the inertial criterion. The inertialess regime corresponds to a balance between viscous forces and the Lorentz force near the wall. The inertial regime corresponds to a balance between inertial forces and the Lorentz force. The goal of our study is to identify and/or clarify which regime dominates as a function of the values of the control parameters. In order to achieve this goal, we adopt an experimental-like approach by varying the injected current $I_{0}$ at the boundaries and the externally imposed magnetic field $B_{0}$. We will limit our study to cases where the magnetic 


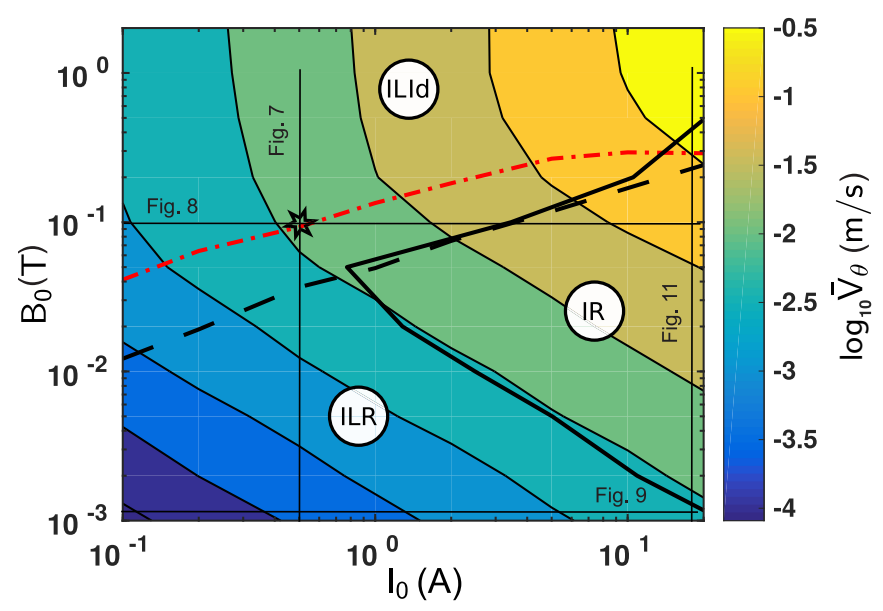

FIG. 5. Mean toroidal flow versus $B_{0}$ and $I_{0}$. The black dashed line corresponds to the critical ratio for the inertial criterion $2 h^{2} \mathrm{Re} / \mathrm{Ha}^{2} \bar{r}^{2}=1$. The black solid line is the numeric estimate of $\langle\|(\mathbf{V} \cdot \nabla) V\|\rangle /\langle\|v \Delta V\|\rangle=$ 0.7. The red dot-dashed line is the numeric estimate of the induction ratio $\tau=0.5$ [Eq. (29)]. There are three clear regimes: the inertialess regime ILId, the inertial regime IR, and the weak regime ILR.

Mach number satisfies

$$
M_{\mathrm{Ma}}=\frac{V_{\theta}}{V_{A}} \lesssim 1
$$

which is convenient if one wants to compare with previous works $[1,2,6,10,13]$. Note, however, that the experimental work of Moresco and Alboussière [7] addresses the issue of large magnetic Mach numbers, and we will return to this point later. In this section, the geometry is that of the reference case.

\section{A. Characterization of flow regimes as a function of $I_{0}$ and $B_{0}$}

The evolution of the mean toroidal velocity for various values of $B_{0}$ and $I_{0}$ is reported in Fig. 5 . The boundary between the inertialess and inertial regimes is given by a balance between the inertial and the viscous terms in the toroidal momentum equation (1), leading to the inertial criterion limit $2 R_{e}=\mathrm{Ha}^{2}(\bar{r} / h)^{2}$, displayed as a dashed black line in Fig. 5. At high magnetic fields $B_{0}$, i.e., above the inertial criterion limit, the amplitude of the toroidal velocity $V_{\theta}$ does not depend on $B_{0}$. At low magnetic fields the toroidal velocity $V_{\theta}$ is proportional to $\left(B_{0} I_{0}\right)^{\alpha}$, where $\alpha>0$ will be specified below.

Nevertheless, the criterion $2 R_{e}=\mathrm{Ha}^{2}(\bar{r} / h)^{2}$ fails in the limit of low currents and/or low magnetic fields, as identified in Fig. 6(a). This occurs when the widths of the boundary layers are a significant fraction of the length of the device, as observed in Fig. 6(a). In order to investigate
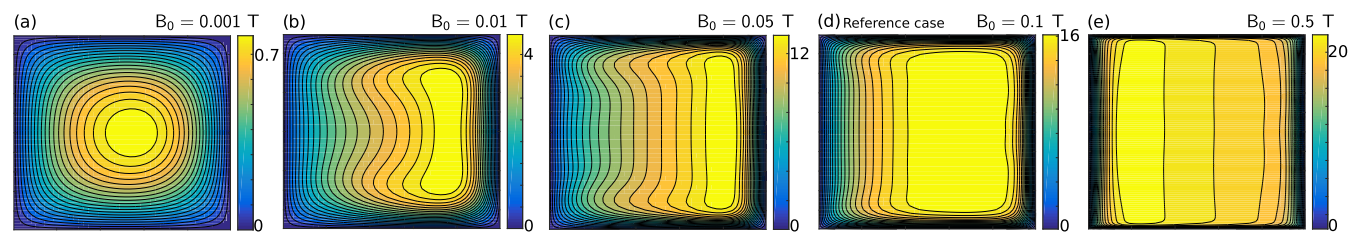

FIG. 6. Mean toroidal flow velocity snapshots for various magnetic fields, from (a) to (e), $B_{0}=$ $0.001,0.01,0.05,0.1,0.5 \mathrm{~T}$. The other parameters are reference case ones. 
whether the regime is dominated by inertia or viscous stress, we define a volume-averaged Reynolds number $\mathcal{R}$ as

$$
\mathcal{R}=\frac{\langle\|(\mathbf{V} \cdot \nabla) V\|\rangle}{\langle\|v \Delta V\|\rangle} .
$$

For high- $B_{0}$ or $-I_{0}$ values, the high- $\mathcal{R}$ criterion matches the inertial criteria previously introduced (the dashed and solid black lines overlap). This is no longer the case at low- $B_{0}$ values, when the width of the boundary layers reaches the size of the device.

Let us now focus on the inertialess regime and introduce a distinction between the regimes observed above and below the black dashed line in Fig. 5, referred to as inertialess-ideal (ILId) and inertialess-resistive (ILR) regimes, respectively, from an analysis of the dominant terms in Ohm's law. At high magnetic fields, when the induction term is important, e.g., when $U \times B_{0} \gg \eta J$, the toroidal magnetic field is expelled from the center of the channel to the top and bottom Hartmann layers, which defines the inertialess-ideal regime. This regime is also described by a depletion of radial current within the core, or equivalently a vanishing vertical gradient of the radial current in the core,

$$
-\frac{\bar{r}}{\sqrt{\mathrm{Pm}}} J_{r}(\bar{r}, h / 2)=\partial_{z} b(\bar{r}, h / 2) \approx 0,
$$

for the inertialess-ideal regime. The resistive regime corresponds to a balance between the resistive term and the radial potential in Ohm's law, e.g., $U \times B_{0} \ll \eta J$. The current is then roughly homogeneously distributed over the height of the channel. Accordingly, $b \propto B_{\theta} / r$ evolves linearly with the height $z$. The current at midheight of the section is then estimated as

$$
\partial_{z} b(\bar{r}, h / 2) \approx \frac{b_{0}-b_{h}}{2}
$$

for the resistive regime. We thus introduce a criterion based on the value of the current in the center of the channel and introduce the induction ratio $\tau$ as

$$
\tau=2 \frac{\partial_{z} b(\bar{r}, h / 2)}{b_{0}-b_{h}} .
$$

The ideal regime is thus defined by $\tau \sim 0$, while $\tau \rightarrow 1$ for strongly resistive regimes. Arbitrarily, we define the transition between both regimes at $\tau=0.5$, which is displayed as a red dot-dashed line in Fig. 5. At low injected current $I_{0}$, the inertialess-ideal (inertialess-resistive) regime is observed above (below) the red dot-dashed line in Fig. 5. Henceforward, we will label these ILId and ILR regimes, respectively. Note that, at low injected current $I_{0}$, the inertial criterion $2 R_{e}=\mathrm{Ha}^{2}(\bar{r} / h)^{2}$ (black dashed line) corresponds to $\tau=0.9$. In Fig. 5 the inertial regime is in fact an inertial-resistive (IR) regime. Note that for higher current and/or magnetic field, we should reach a fourth regime on the right of the graph, an inertial-ideal (IId) regime.

Let us specify that the ILId regime corresponds to the first regime historically studied with the experiments and calculations of Baylis and Hunt [1,25]; the IR regime was also described in these pioneering works and further investigated more recently [2]. The regimes reached in the experimental work of Moresco and Alboussière [7] will be discussed in Sec. V B.

\section{B. Scaling laws}

As can be seen in Fig. 5, our reference case (black star) lies in the ILId regime, but is very close to the other two regimes. In this section we aim to obtain and/or verify the scaling laws characterizing the different regimes by varying the control parameters from the reference case. Figures 7 and 8 show the evolution of $V_{\theta}$ with $B_{0}$ and $I_{0}$, respectively. In this subsection we compare the numerical results with theoretical scaling laws in the rest of this section. 


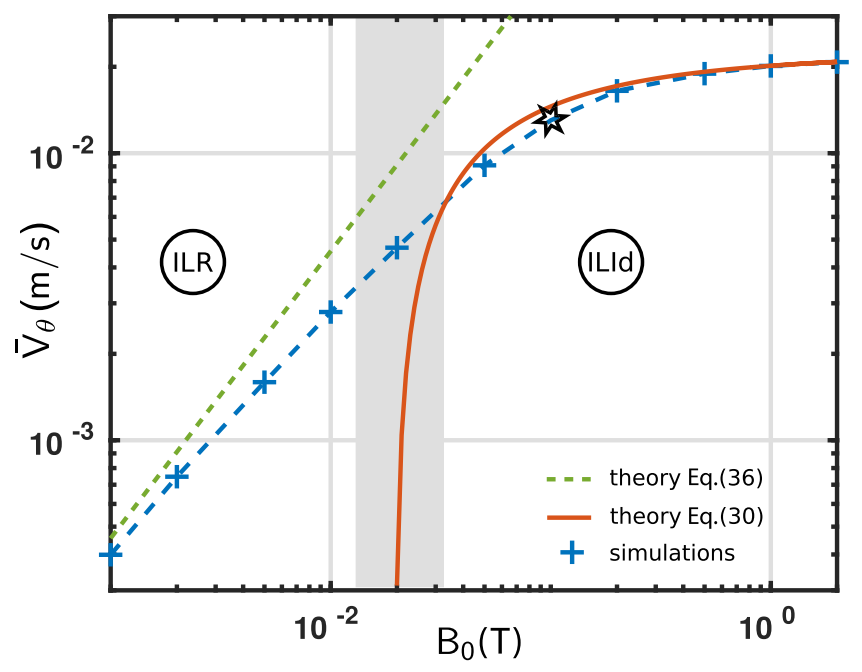

FIG. 7. Mean toroidal flow versus imposed magnetic field. The other parameters are reference case parameters. The gray zone separates the limit of validity of the ILId and ILR regimes. The orange line shows the theoretical ILId regime and the green line the theoretical ILR regime.

\section{Inertialess-ideal regime}

We recall here the results of Baylis and Hunt [1,25], in which the toroidal velocity in the inertialess regime is experimentally and analytically characterized. In this early work, fluctuations of the poloidal fields are neglected, which corresponds to $w=\psi=0$ in our notation. The toroidal velocity is then expressed as (in $\mathrm{m} / \mathrm{s}$ )

$$
V_{\theta}^{\mathrm{ILId}}=\frac{I_{0} \ln \left(r_{1} / r_{0}\right)}{4 \pi\left(r_{1}-r_{0}\right)} \sqrt{\frac{\eta}{\rho \nu}}\left[1-\frac{2}{\mathrm{Ha}}-\frac{0.956 \sqrt{2} h \ln \left(r_{0} / r_{1}\right)}{2 \sqrt{\mathrm{Ha}}}\left(\frac{1}{r_{1}}+\frac{1}{r_{0}}\right)\right] .
$$

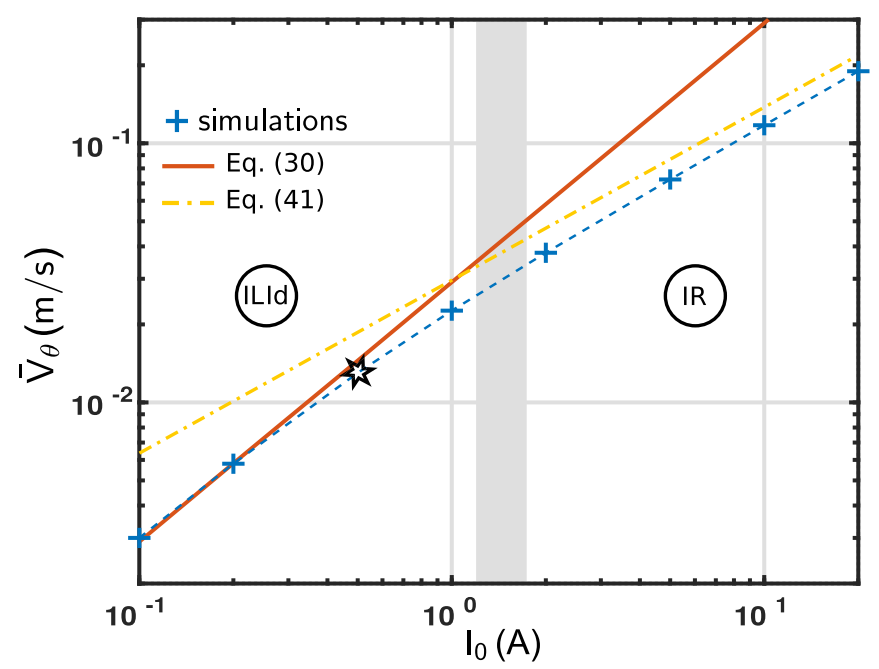

FIG. 8. Mean toroidal flow versus imposed current. The orange line shows the theoretical mean $V_{\theta}$ for the ILId regime [Eq. (30)] and the yellow dot-dashed line the theoretical mean $V_{\theta}$ for the IR regime [Eq. (41)]. The gray zone separates the limit of validity of the ILId and IR regimes. 
The last two terms on the right-hand side are corrections for radially narrow ducts where the Shercliff layer $\delta_{\text {Sh }}$ becomes comparable to $\bar{r}$ or, equivalently, when the magnetic field is not strong enough. It is enlightening and straightforward to extract the dominant term of this formula. Indeed, in such a regime, as already discussed, the radial current is expelled towards the Hartmann layers. Considering that all the current concentrates uniformly in these layers of total axial width $2 \mathrm{~h} / \mathrm{Ha}$ and localized at $\bar{r}$, an estimate of the current density in the layers is

$$
j \simeq \frac{I_{0} \mathrm{Ha}}{2 \pi \bar{r} 2 h} .
$$

The toroidal velocity is then obtained from the balance between the diffusion term and the Lorentz force in the Hartmann layers (in $\mathrm{m} / \mathrm{s}$ )

$$
V_{\theta}^{\mathrm{ILId}} \propto \frac{I_{0}}{4 \pi \bar{r}} \sqrt{\frac{\eta}{\rho \nu}} \propto \frac{I_{0} \ln \left(r_{1} / r_{0}\right)}{4 \pi\left(r_{1}-r_{0}\right)} \sqrt{\frac{\eta}{\rho \nu}},
$$

using $r_{0} \lesssim r_{1}$. This expression is consistent with Eq. (30). Note that it gives the scaling law for the current and the geometric factor but not for the magnetic field which appears in higher-order terms. The prediction from Eq. (30) is displayed as an orange solid line in Figs. 7 and 8. Numerical and theoretical results agree well for $B_{0}>0.03 \mathrm{~T}$, as can be observed in Fig. 5. As expected, for lower magnetic fields, Eq. (30) does not agree with the numerical results.

\section{Inertialess resistive regime}

In the inertialess-resistive regime, inertia is neglected in Eq. (1) and the induction term is neglected in Ohm's law (2). In this regime, the radial current density is constant over the duct in a first approximation (neglecting curvature, i.e., when $\bar{r} \gg \Delta r$ ),

$$
j=\frac{I_{0}}{2 \pi r h} \simeq \frac{I_{0}}{2 \pi \bar{r} h} .
$$

The steady state of Eq. (1) correspond to the balance between the Lorentz force and the viscous stress: $j_{r} B_{0} \simeq-\rho \nu \Delta V_{\theta}$. We recall here that in this regime, the width of the boundary layers is comparable to the channel dimensions. Thus, one obtains

$$
\Delta V_{\theta}(r, z)=-\frac{I_{0} B_{0}}{2 \pi \bar{r} h \rho \nu} .
$$

Figure 6(a) shows a numerical solution of $V_{\theta}$ in this regime; the isovalues have a nearly circular shape. With a circular section $\tilde{r}=\sqrt{(r-\bar{r})^{2}+z^{2}}$, one can express the toroidal flow profile from the integration of Eq. (34),

$$
V_{\theta}(\tilde{r})=\frac{I_{0} B_{0}}{8 \pi \bar{r} h \rho \nu}\left(\tilde{r}_{e}^{2}-\tilde{r}^{2}\right)
$$

with $\tilde{r}_{e}$ the effective radius of the channel. The boundary conditions are $V_{\theta}^{\prime}(\tilde{r}=0)=0$ and $V_{\theta}\left(\tilde{r}_{e}=\right.$ $0)=0$. The channel section is constant with $\tilde{r}_{e}=\sqrt{\Delta r h / \pi}$. One obtains for the mean toroidal flow in the ILId regime, after a volume integration (in $\mathrm{m} / \mathrm{s}$ ),

$$
V_{\theta}^{\mathrm{ILR}}=\frac{I_{0} B_{0} \Delta r}{16 \pi^{2} \bar{r} \rho \nu}
$$

The evolution of $V_{\theta}^{\mathrm{ILR}}$ predicted from Eq. (36) is displayed as a green dashed line in Fig. 7, and good agreement with numerical simulations is observed. To assess the robustness of this prediction, the evolution of $V_{\theta}$ with the injected current for a given low magnetic field of $1 \mathrm{mT}$ is reported in Fig. 9. In this case, the inertial regime starts for injected currents higher than $20 \mathrm{~A}$. Once again, good agreement between the numerical results and the predicted values $V_{\theta}^{\mathrm{ILR}}$ is observed. Note that this regime is easily obtained using conductive aqueous solutions of $\mathrm{CuSO}_{4}(\eta=1.33 \Omega \mathrm{m})[14,15]$. 


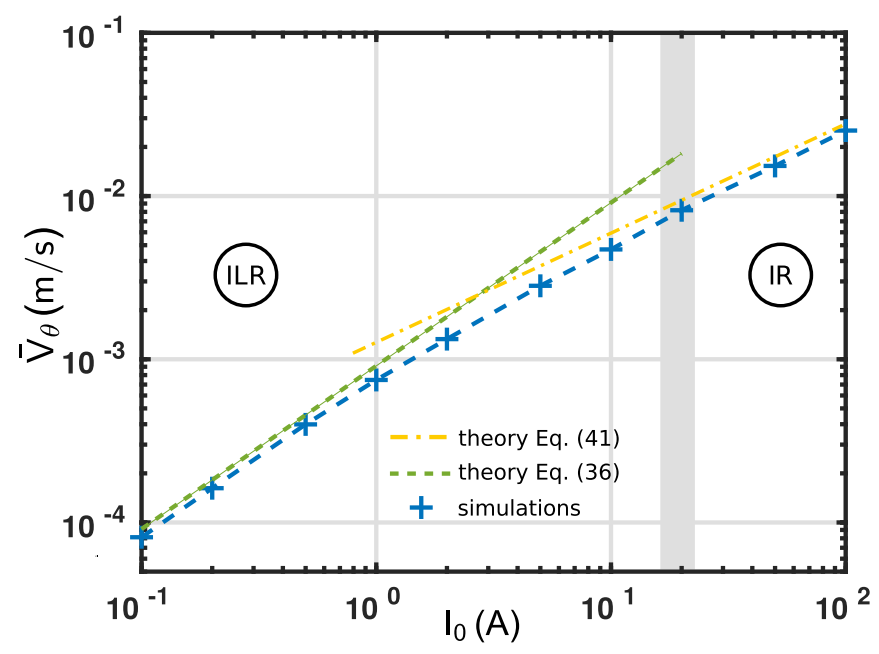

FIG. 9. Mean toroidal flow for the reference case with $B_{0}=1 \mathrm{mT}$ and with various current values, obtained from simulation (blue pluses). The scan crosses the weak regime ILR to the inertial regime IR around $I_{0}=$ $20 \mathrm{~A}$. The theoretical value (36) in the ILR regime is shown by the green line and the theoretical value (41) in the IR regime is shown by the yellow dot-dashed line.

\section{Inertial resistive regime}

In this regime, the induction term $V \times B$ can also be neglected in Ohm's law and the current is uniformly distributed in toroidal sections; Eq. (33) is thus valid. This regime is also characterized by a balance between inertia and the Lorentz force for the momentum equation (1) projected in the toroidal direction, e.g., $V_{r} \partial_{r} V_{\theta}$ balances the Lorentz force in the core of the channel sincelg

$$
\frac{\mathcal{O}\left(V_{z} \partial_{z} V_{\theta}\right)}{\mathcal{O}\left(V_{r} \partial_{r} V_{\theta}\right)} \approx \frac{\partial_{r} w \partial_{z} u}{\max \left(\partial_{z} w \partial_{r} u, u \partial_{z} w / r\right)} \ll 1
$$

Indeed, the presence of Dean's rolls ensures $\mathcal{O}\left(\partial_{r} w\right) \simeq \mathcal{O}\left(\partial_{z} w\right)$ [see Fig. 10(c)]. In this regime, the field $u$ has almost no $z$ gradient [see Fig. 10(a)], and thus $\left|\partial_{z} u\right| \ll\left|\partial_{r} u\right|$. Moreover, the characteristic length of the $r$ gradient is $\Delta r \ll \bar{r}$. It follows that, as already discussed [2], the dominant convective term is $r^{-2} u \partial_{z} w=V_{r} V_{\theta} / r$, which is the Coriolis acceleration due to the curvature of the channel. Thus, in the core of the channel

$$
\rho \frac{V_{r} V_{\theta}}{\Delta r} \simeq \frac{I_{0} B_{0}}{2 \pi \bar{r} h}
$$

This advection term includes $V_{r}$ and involves thus the radial momentum equation balance. The drive of this equation is the centripetal acceleration, the $V_{\theta}^{2} / \bar{r}$ term which arises explicitly in cylindrical coordinates.

We now introduce the terms $V_{r}^{-}$and $V_{\theta}^{-}$to denote the order of magnitude of the radial and toroidal velocities, respectively, in the viscous layers. Large convective rolls (or Dean vortices) connect the radial flow velocity $V_{r}$ in the core to the one, $V_{r}^{-}$, in the horizontal viscous layers. The latter are generated by the strong axial gradient of the toroidal velocity along the wall. They are also bounded by the Dean vortices which advect the fluid up and down from the center. These rolls have a vertical height $f$ bounded by half of the channel's height $h / 2$. As can be seen in Fig. 10(c), they are compressed toward the top and bottom walls. Thus, an estimate of the viscous layer width between the core of the vortex and the wall is $\delta_{v}=\sqrt{2 \pi \bar{r} v / V_{\theta}^{-}}$. As expected, our simulations show a linear spatial evolution of the toroidal velocity profile in this boundary layer. The measure of $\delta_{v}$ 

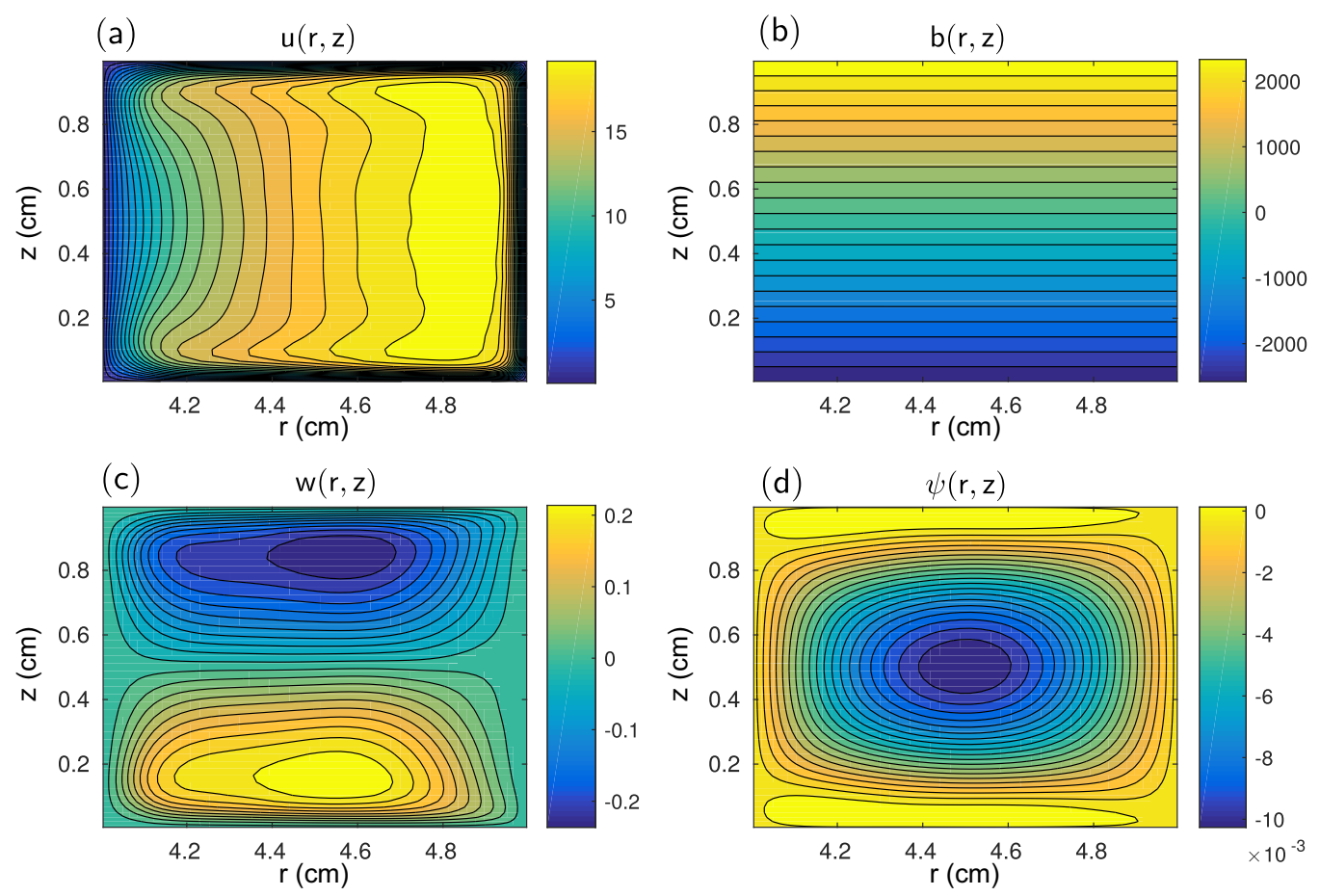

FIG. 10. Plot of the $(r, z)$ cross section of the flow and the current (a) $u(r, z),(\mathrm{b}) b(r, z),(\mathrm{c}) w(r, z)$, and (d) $\psi(r, z)$ for the reference case with $B_{0}=1 \mathrm{mT}$ and $I_{0}=100 \mathrm{~A}$ in the inertial regime $\left(\mathrm{Pm}=1.48 \times 10^{-7}\right.$ and $\mathrm{Ha}=0.26)$.

corresponds to the distance between the wall and the core of the vortex. We thus estimate

$$
V_{\theta}^{-} \simeq \frac{V_{\theta}}{2}
$$

We emphasize that the toroidal velocity is laminar outside the viscous layer and has almost no axial gradient except in those layers. It follows, from the incompressibility of the flow, that

$$
f h V_{r}=2 \delta_{v} V_{r}^{-}
$$

showing that, roughly, the top vortex advects from the core up to the top walls, a quarter of the core flow $\left(\delta_{v} \ll h / 4\right)$. Due to symmetry, the same arguments apply for the bottom roll. Concerning the perpendicular flow, there are narrow recirculation layers where the centripetal force balances viscous stress

$$
\frac{V_{\theta}^{-2}}{\bar{r}} \simeq v \frac{V_{r}^{-}}{L_{D}^{2}}
$$

where $L_{D}=\sqrt{\frac{h v}{2 V_{\theta}^{-}}}$. Note that we have introduced a second viscous width $\delta_{v} \neq L_{D}$ because the force balance is in the gradient localization of the viscous layers. Let us emphasize that the boundary layers are viscous layers, not depending on the Hartmann number, since in this regime the flow is assumed strong and the magnetic field weak. Thus, there is no concentration of current and flow in narrow Hartmann layers [see Fig. 10(b)]. Gathering the three previous equations, we get the scaling 


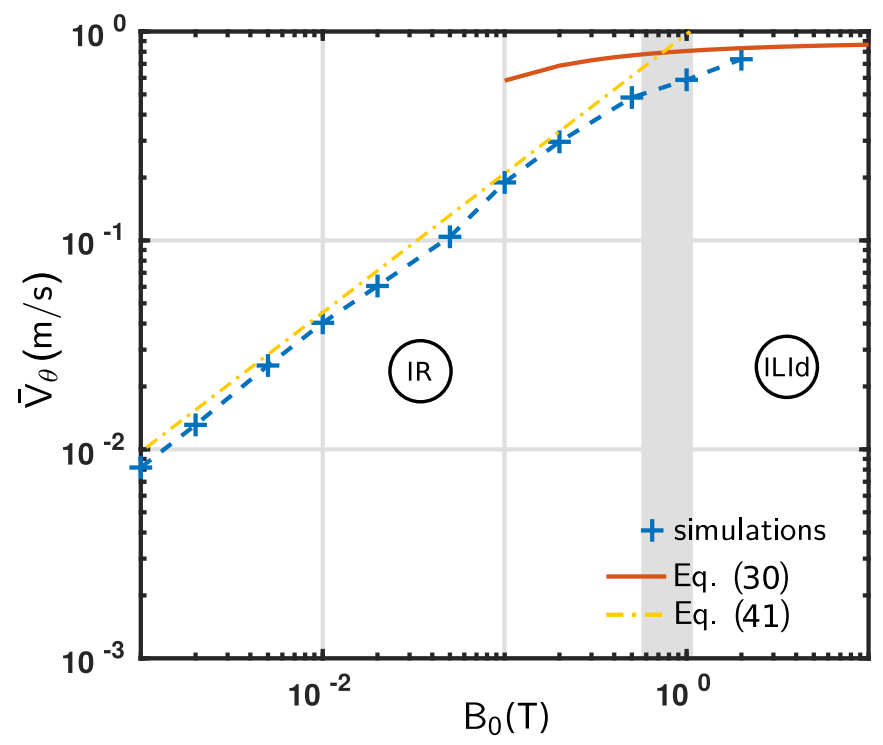

FIG. 11. Mean toroidal flow for the reference case with $I_{0}=20 \mathrm{~A}$ and with various magnetic field values, obtained from simulation (blue pluses). The scan crosses the inertial regime IR to the inertialess regime ILId around $B_{0}=1 \mathrm{~T}$. The theoretical value (30) in the ILId regime is shown by the orange line and the theoretical value (41) in the IR regime is shown by the yellow dot-dashed line.

law in the inertial regime (in $\mathrm{m} / \mathrm{s}$ )

$$
V_{\theta}^{\mathrm{IR}} \simeq\left(\frac{f I_{0} B_{0} \Delta r}{2 \pi \rho}\right)^{2 / 3}\left(\frac{4}{\pi \bar{r} h^{2} v}\right)^{1 / 3} .
$$

The evolution of this prediction as a function of $I_{0}$ is shown in Figs. 8 and 9 and as a function of $B_{0}$ in Fig. 11. We observe good agreement of this relation with the numerical results.

\section{Influence of the geometry}

Historically, the experimental ducts $[5,8]$ had a square geometry $\epsilon=h / \Delta r=1$ with mean radii (in $\mathrm{cm}$ ) $\bar{r} \in 5.4,6.8$ and heights (in $\mathrm{cm}$ ) $h \in 0.4,3$.1. The results introduced previously in this article have also been obtained using a square cross section. However, one may expect that the regimes will depend on the geometric parameters. Indeed, as shown in Fig. 12, the amplitude and profile of $V_{\theta}$ significantly depend on the aspect ratio. The points correspond to simulations of the reference case with varying $\varepsilon$ [Fig. 12(a)] and $\bar{r}$ [Fig. 12(b)]. The regimes are again identified by assessing the induction ratio value (29) and the integral $\mathcal{R}=\langle\|(\mathbf{V} \cdot \nabla) V\|\rangle /\langle\|v \Delta V\|\rangle$. The theoretical predictions presented above are also reported in Fig. 12. Note that the previous calculations have to be slightly modified to the case of tall tori. For the ILR regime, the assumption $\Delta r \ll \bar{r}$ is replaced by $\partial_{z} \simeq 0$. Equation (34) becomes

$$
\partial_{r}^{2} V_{\theta}(r)+\frac{1}{r} \partial_{r} V_{\theta}(r)-\frac{1}{r} V_{\theta}(r) \simeq-\frac{I_{0} B_{0}}{2 h \pi \rho \nu r}
$$

The boundary conditions are $V_{\theta}\left(r_{0}\right)=V_{\theta}\left(r_{1}\right)=0$. The value averaged in the complete volume is (in $\mathrm{m} / \mathrm{s}$ )

$$
V_{\theta}^{\mathrm{ILR}}=\frac{I_{0} B_{0} \bar{r}}{8 \pi h \rho v}\left[1-\left(\frac{r_{0} r_{1}}{\Delta r \bar{r}} \ln \left(r_{1} / r_{0}\right)\right)^{2}\right] .
$$



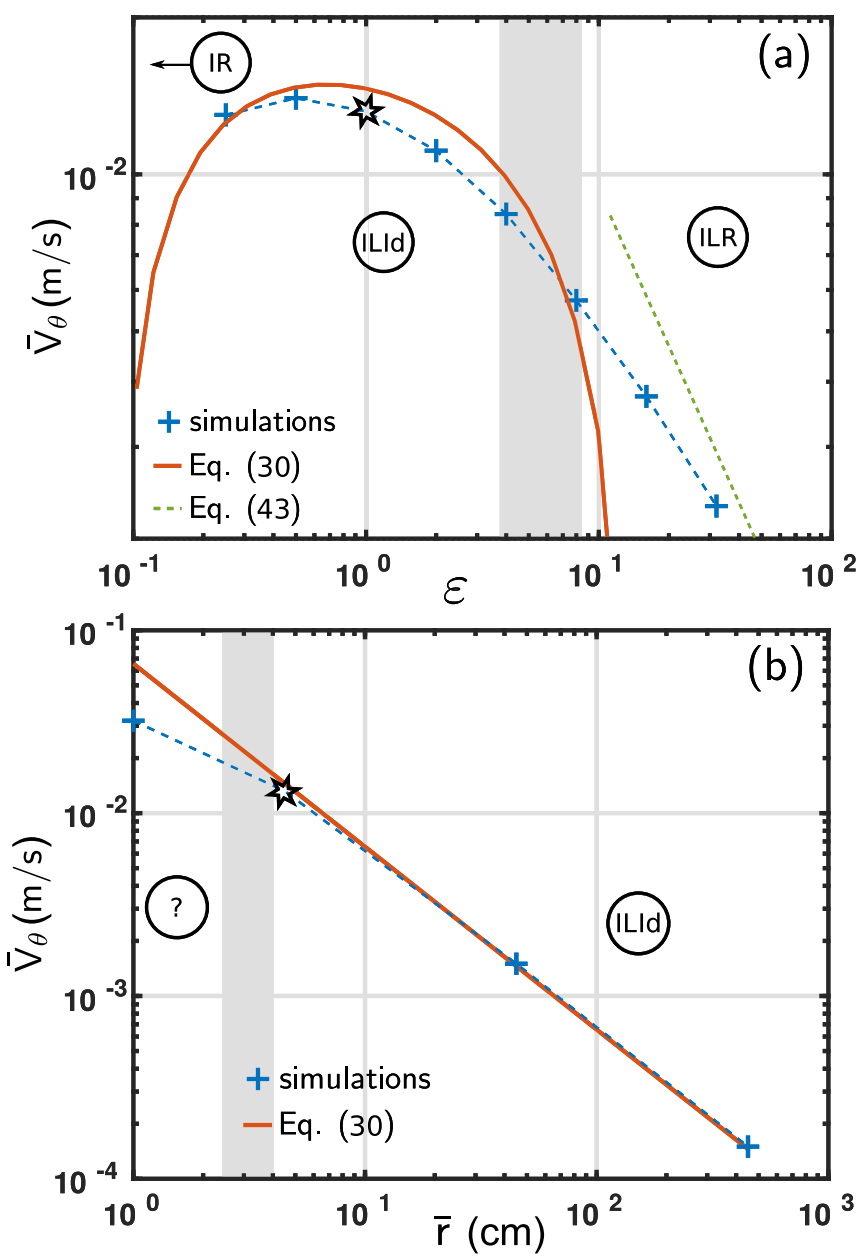

FIG. 12. Mean toroidal flow for the reference case with (a) various aspect ratio values and (b) various mean radii. Blue pluses show the results obtained from simulation and the lines are for theories. The scan crosses the identified regimes. The theoretical value (30) in the ILId regime is shown by the orange line.

The ILId regime does not depend on the height of the torus, according to Eq. (30); the height $h$ appears explicitly only in a corrective term. However, we observe in Fig. 12(a) that this is not true for large $\epsilon$ or $h$. Indeed, the existence of Hartmann and Shercliff layers is one of the assumptions of the calculation. In the ILId regime, the Shercliff layer disappears when $h / \sqrt{\mathrm{Ha}}>\Delta r$, thus for a torus taller than $25 \mathrm{~cm}$ in our reference case. The absence of Shercliff layers prevents the current from being expelled to the top and bottom of the duct; the current field lines are thus along radii. For large enough $\epsilon$, the induction term may thus be neglected and the system enters a resistive regime. Moreover, top and bottom Hartmann layers do not depend on the height $h$. As explained in Sec. III B, they prevent strong recirculating flows and maintain the inertialess character of the regime when increasing $\epsilon$. We thus have an ILR regime for tall tori. Note that, on the contrary, for a flat torus or when decreasing $\epsilon$, Hartmann layers overlap and disappear if $1 / \mathrm{Ha}>1(h<0.5 \mathrm{~mm})$. We did not investigate numerically this regime.

Increasing the mean radius of the torus $\bar{r}$ decreases the radial current density, since the total current is injected across a larger surface. The predictions of the ILId regime remain valid, as observed in Fig. 12(b). 


\section{COMPARISON WITH EXPERIMENTS}

\section{A. Tall torus}

Let us first focus on the experimental results reported by Boisson et al. [6,13], in which the toroidal velocity was interpreted to evolve as $V_{B}=2.1 \sqrt{\frac{I_{0} B_{0} \Delta r}{r_{1} r_{0} \rho}}-0.015 \mathrm{~m} / \mathrm{s}$. We provide a comparison with their experimental results obtained using galinstan. Simulations are done with their experimental parameters: $r_{0}=1 \mathrm{~cm}, r_{1}=4 \mathrm{~cm}, h=12 \mathrm{~cm}, \eta=2.89 \times 10^{-7} \Omega \mathrm{m}, \rho=$ $6440 \mathrm{~kg} \mathrm{~m}^{-3}$, and $v=3.73 \times 10^{-7} \mathrm{~ms}^{-2}$. The magnetic field is varying from 0.01 to $0.1 \mathrm{~T}$ and the current from $1 \mathrm{~mA}$ to $20 \mathrm{~A}$.

Results from our simulations are compared to the scaling law $V_{B}$ proposed in Ref. [13] and displayed in Fig. 13. Both the maximum value and the spatial average of the toroidal velocity are displayed in Fig. 13. Figure 13(a), in linear scale, shows good agreement between the scaling law $V_{B}$ and the maximum toroidal velocity observed in our simulations. This is consistent with the experimental procedure from Ref. [13], where the average is done along a $z$ line at a radial position where the toroidal flow reaches its maximal value. This average overestimates the value of the volume average (see circles in the figure). Figure 13(b), in logarithmic scale, allows us to assess the scaling laws derived in the present article. The scaling law for the ILId regime curve is shown for $B=0.1 \mathrm{~T}$ (red line) and fits well the corresponding data. As the magnetic field decreases, a transition towards the ILR regime is observed, with good agreement between the low-magnetic-field data and the scaling law for the ILR regime (black dashed line). The last regime IR corresponds to high injected currents (and thus to high- $I_{0} B_{0}$ values). Good agreement is observed between numerical data and theoretical predictions for this regime. Note that, for tall geometries, Dean's rolls are located on the very top and bottom of the duct, and thus $f \rightarrow 1$ in Eq. (39). This analysis shows that the transition between the inertial regime and the inertialess regimes may not be sufficiently well resolved when using the parameter control $I_{0} B_{0}$ and that both the inertialess-ideal and the inertialess-resistive regimes may indeed have been observed in Ref. [6].

\section{B. Square torus}

Let us now investigate the evolution of the mean toroidal flow $V_{\theta}$ with the imposed magnetic field and the injected current in the device described in Fig. 1, as reported in detail by Moresco and Alboussière [8] and reproduced in Fig. 14. The friction factor (ratio between the Lorentz force and the radial component of the inertial force at the center) is defined as $F_{\mathrm{Alb}}=\frac{I_{0} B_{0}}{V_{\theta}^{2} \rho 2 \pi \bar{r}}$, with $\bar{r}=$ $\left(r_{1}+r_{0}\right) / 2$, while the Hartmann and Reynolds numbers are defined as $R_{\mathrm{Alb}}=\mathrm{Re} / \mathrm{Ha}=\frac{L V_{\theta}}{v} \frac{\sqrt{\rho \eta v}}{L B_{0}}$. The experimental data (thin black pluses) reported in Fig. 1 gather in regimes where the injected current and magnetic field range from 1 to $400 \mathrm{~A}$ and from 1 to $13 \mathrm{~T}$, respectively [8]. In this experiment the Shercliff layers are very small $\left[\delta_{\mathrm{Sh}} /\left(r_{1}-r_{0}\right)\right.$ ranges between 0.017 and 0.062$]$, and the corrective terms are thus negligible in Eq. (30). Here, owing to numerical limitation, we have used weaker magnetic fields for the numerical investigation (up to $1 \mathrm{~T}$ ) to obtain regimes in the ILId and IR regimes. The evolution according to Eq. (30) (the colored dashed lines in Fig. 14 for each value of the magnetic field) is compared to our numerical simulations (colored symbols). We also investigated a weak magnetic field of $1 \mathrm{mT}$, for which the flow lies mostly in the ILR regime according to Eqs. (36) and (41). The theoretical predictions, experiments, and numerical simulations match correctly. A peculiar feature of the experimental results [8] is the bifurcation observed at $R_{\mathrm{Alb}} \simeq 400$; the bifurcation occurs at a given point $\left(R_{\mathrm{Alb}, c}, F_{\mathrm{Alb}, c}\right) \approx\left(400,5 \times 10^{-3}\right)$ in the diagram $\left(R_{\mathrm{Alb}}, F_{\mathrm{Alb}}\right)$ whatever the values of $B_{0}$ and $I_{0}$. This observation motivated several studies [12,26-31]. In short, this is linked to a bifurcation from an essentially 2D dynamics $(r-z)$, including instabilities to a 3D dynamics. The good agreement between experimental, numerical, and theoretical results [according to Eq. (30)] at $R_{\mathrm{Alb}}<R_{\mathrm{Alb}, c}$ illustrates that the regime is the inertialess-ideal regime. The bifurcation observed experimentally develops from the ILId regime; thus we can determine the location of the bifurcation in the $\left(I_{0}, B_{0}\right)$ parameter space, displayed as a blue dotted line in Fig. 15 . 

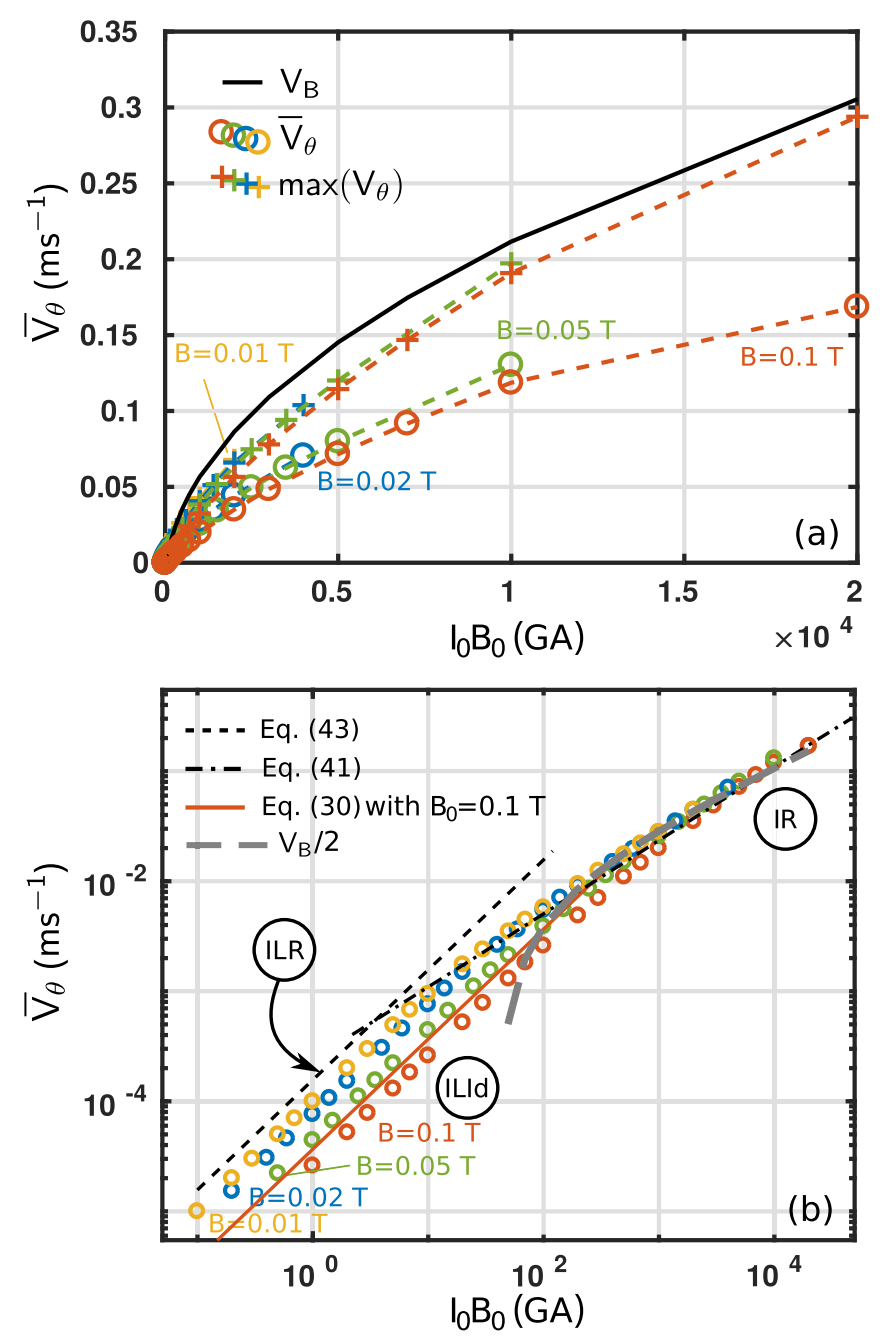

FIG. 13. Values of toroidal flow versus $I_{0} B_{0}$. The black solid line shows the scaling of Boisson et al. [6,13]. The pluses are the maximum values of the toroidal flow and the circles are the mean values. Colors stand for the magnetic field: yellow, $10 \mathrm{mT}$; blue, $20 \mathrm{mT}$; green, $50 \mathrm{mT}$; and orange, $0.1 \mathrm{~T}$. The parameters are $r_{0}=1 \mathrm{~cm}$, $r_{1}=4 \mathrm{~cm}, h=12 \mathrm{~cm}$, galinstan, $\eta=2.89 \times 10^{-7} \Omega \mathrm{m}, \rho=6440 \mathrm{~kg} \mathrm{~m}^{-3}, v=3.73 \times 10^{-7} \mathrm{~ms}^{-2}, B_{0}$ from $10 \mathrm{mT}$ to $0.1 \mathrm{~T}$, and $I_{0}$ from $1 \mathrm{~mA}$ to $20 \mathrm{~A}$. (a) Linear scale. (b) Logarithmic scale with the theoretical value of Eq. (43) (black dashed line), Eq. (30) (orange solid line for $B_{0}=1 \mathrm{~T}$ ), and Eq. (41) (black dot-dashed line). Shown in gray is $V_{B}$ divided by 2 to adapt the measurements from maximal values to averages.

We observe that the transition seems to correspond to the extension of the black solid line which separates the inertialess and inertial zones. Taking into account also the induction ratio criterion (red dot-dashed line) which separates the resistive and ideal regimes, we can infer that the observed bifurcation also corresponds to a transition from an inertialess-ideal regime to an inertial-ideal one, the transition occurring for large enough current.

To summarize, the bifurcation observed by Moresco and Alboussière is associated with a change of the dimensionality of the turbulence, from two to three dimensions, and to the onset of the inertial flow. The correlation of those two effects suggests that it is the advective operator which is responsible of this generation of the third dimension in the turbulence. However, important 


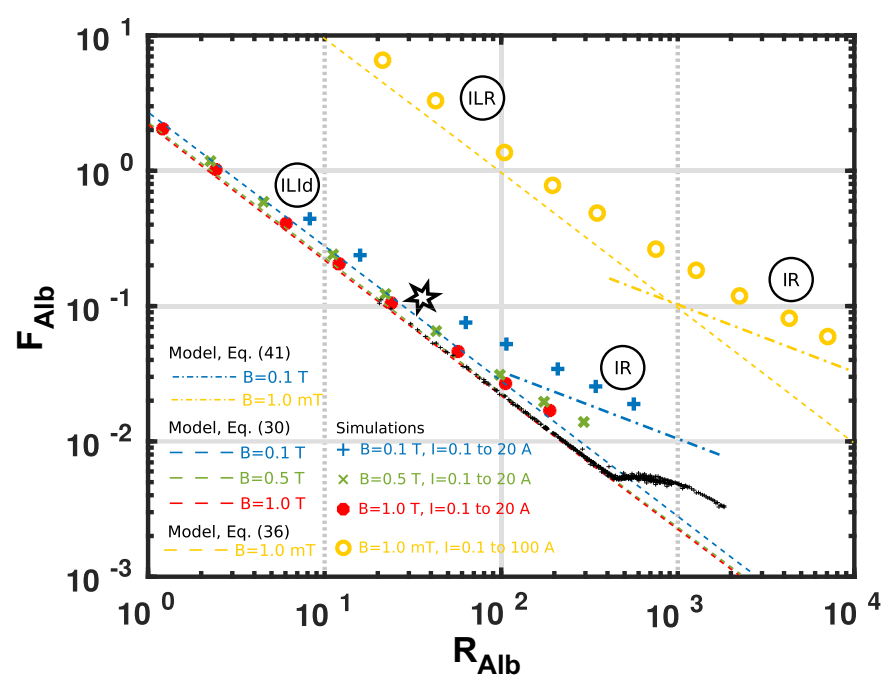

FIG. 14. Friction factor $F_{\mathrm{Alb}}$ as a function of $R_{\mathrm{Alb}}$. The thin black pluses show the experimental measurements (1-13 T and 10-400 A); blue pluses the simulations with $B_{0}=0.1 \mathrm{~T}$ and $I_{0}=0.1-20 \mathrm{~A}$; green crosses the simulations with $B_{0}=0.5 \mathrm{~T}$ and $I_{0}=0.1-20 \mathrm{~A}$; closed red circles the simulations with $B_{0}=1 \mathrm{~T}$ and $I_{0}=0.1-20 \mathrm{~A}$; open yellow circles the simulations with $B_{0}=1 \mathrm{mT}$ and $I_{0}=0.1-100 \mathrm{~A}$; dashed lines the theoretical values calculated with Eq. (30) for blue, green, and red and with Eq. (36) for yellow; and dot-dashed lines the theoretical values calculated with Eq. (41). The black star denotes the reference case.

questions remain on this transition. According to Fig. 14, the experimental measurement of the $V_{\theta}$ scaling law does not seems to agree with the one of the ILId regime $\left[V_{\theta} \propto\left(I_{0} B_{0}\right)^{2 / 3}\right]$. We list some possible scenarios. First, the scaling law measured could be the one of the fourth regime, the IId regime. Second, the 3D turbulence could strongly modify the steady state obtained in axisymmetric simulations. Third, the transition observed by Moresco and Alboussière could consist of several transitions. For example, it is known in hydrodynamics that there are many inertial regimes with various recirculation patterns [32]; it can be the same in this setup. The extension of the present work to nonaxisymmetric flow is in this light an interesting perspective.

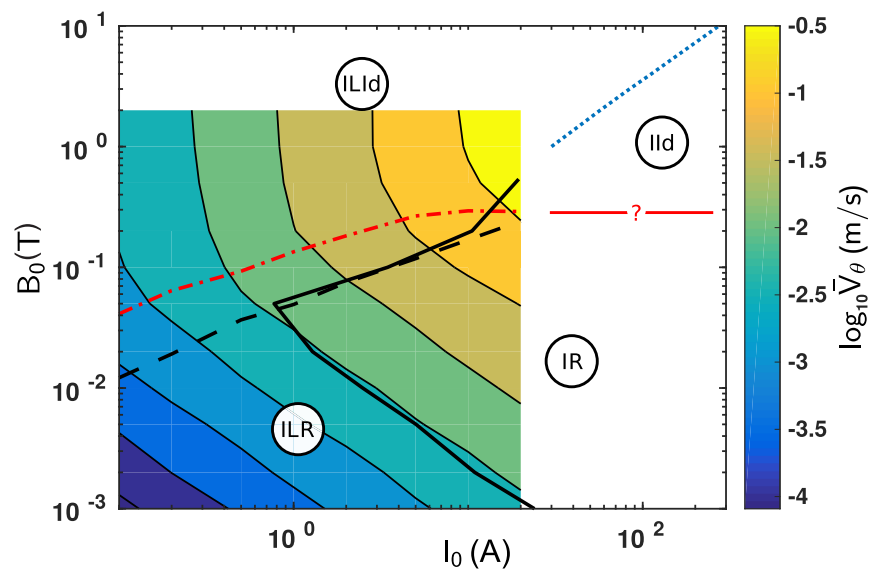

FIG. 15. Same as in Fig. 5 plus extrapolation from experiment (blue dotted line) and a question mark for the limit of the ideal or resistive Ohm law (red dot-dashed line). 


\section{CONCLUSION}

We performed a study of the annular flow driven by an electric field. We showed that even if the asymptotic flow is turbulent, it has a statistical steady state which is comparable to the results obtained from theoretical time-independent solutions. We explored the flow properties by varying four physical parameters: the injected current, the magnetic field, the aspect ratio of the duct, and its mean radius. The results demonstrate the intricate interplay of those parameters causing different effects which determine the flow regimes. They show that the usual classification of the flow in inertial or inertialess regimes does not encompass all the possible regimes. More precisely, we have identified numerically three regimes and verified the power laws which characterize their statistical steady states. It appears that, associated with the presence or absence of Hartmann and Shercliff layers and the relative importance of nonlinear terms, it is difficult to classify the regimes with a few adimensional numbers. As experiments have various designs, we have also investigated the influence of the geometric parameters and found that they impact the nature of the regimes. Finally, taking the parameters of two representative experiments, we have found that the three regimes are present. We have specifically compared our numerical and analytical results with their experimental results. We have recovered the mean toroidal flow values obtained experimentally and highlighted the validity of the data with the power laws of the three regimes. Concerning the experiments of Moresco and Alboussière, we found that the regime change they identified by plotting the friction factor versus the Hartmann and Reynolds numbers is likely to correspond to a crossover from the inertialess-ideal regime to an inertial regime. Such activation of the advection terms is in accordance with the apparition of 3D turbulence.

The value and shape of the toroidal velocity profile are key parameters to control MRI experiments. They require a decreasing angular momentum profile. However, the path to the MRI is still not understood. First, there is still an unobserved regime in our parameter range, the inertial-ideal regime. Whether or not it may produce a favorable toroidal flow in the MRI context is thus open. Second, the MRI is not the only instability that can be triggered with a decreasing angular toroidal momentum profile. Curvature or hydrodynamic instabilities can be triggered in such a context (Rayleigh stability criterion). In our simulations, the Rayleigh criterion is never satisfied in the vicinity of the outer boundary. Suslov et al. [15], by studying an electrolyte flow in a cylinder layer, obtained the same conclusion. Thus, experimental conditions which could avoid this curvature instability must be further investigated to sort out a MRI experiment design. Third, in duct experiments, the poloidal flow was observed experimentally [13]. It seems that this flow corresponds to Dean rolls in inertial regimes [33]. In our study, we observe that they are linked to the angular momentum profiles. They deserve a separate study. Indeed, in hydrodynamics, those secondary flows present a high number of shapes and regimes [32] and lead to various inertial regimes and momentum profiles. Thus, one may also expect such a diversity in magnetohydrodynamic inertial regimes.

\section{ACKNOWLEDGMENTS}

This work has been supported by the French National Research Agency through Jeune Chercheuse/Jeune Chercheur Contract No. ANR-13-JS04-0003-01. This work was granted access to the HPC resources of the FLMSN, "Fédération Lyonnaise de Modélisation et Sciences Numériques", partner of Equipex Equip@meso.

[1] J. A. Baylis and J. C. R. Hunt, MHD flow in an annular channel: Theory and experiment, J. Fluid Mech. 48, 423 (1971).

[2] I. V. Khalzov, A. I. Smolyakov, and V. I. Ilgisonis, Equilibrium magnetohydrodynamic flows of liquid metals in magnetorotational instability experiments, J. Fluid Mech. 644, 257 (2010). 
[3] F. Stefani, T. Gundrum, G. Gerbeth, G. Rüdiger, M. Schultz, J. Szklarski, and R. Hollerbach, Experimental Evidence for Magnetorotational Instability in a Taylor-Couette Flow under the Influence of a Helical Magnetic Field, Phys. Rev. Lett. 97, 184502 (2006).

[4] D. R. Sisan, N. Mujica, W. A. Tillotson, Y.-M. Huang, W. Dorland, A. B. Hassam, T. M. Antonsen, and D. P. Lathrop, Experimental Observation and Characterization of the Magnetorotational Instability, Phys. Rev. Lett. 93, 114502 (2004).

[5] J. A. Baylis, Detection of the onset of instability in a cylindrical magnetohydrodynamic flow, Nature (London) 204, 563 (1964).

[6] J. Boisson, A. Klochko, F. Daviaud, V. Padilla, and S. Aumaître, Travelling waves in a cylindrical magnetohydrodynamically forced flow, Phys. Fluids 24, 044101 (2012).

[7] P. Moresco and T. Alboussière, Weakly nonlinear stability of Hartmann boundary layers, Eur. J. Mech. B 22, 345 (2003).

[8] P. Moresco and T. Alboussière, Experimental study of the instability of the Hartmann layer, J. Fluid Mech. 504, 167 (2004).

[9] Z. Stelzer, S. Miralles, D. Cébron, J. Noir, S. Vantieghem, and A. Jackson, Experimental and numerical study of electrically driven magnetohydrodynamic flow in a modified cylindrical annulus. II. Instabilities, Phys. Fluids 27, 084108 (2015).

[10] Z. Stelzer, D. Cébron, S. Miralles, S. Vantieghem, J. Noir, P. Scarfe, and A. Jackson, Experimental and numerical study of electrically driven magnetohydrodynamic flow in a modified cylindrical annulus. I. Base flow, Phys. Fluids 27, 077101 (2015).

[11] P. Tabeling, Sequence of Instabilities in Electromagnetically Driven Flows between Conducting Cylinders, Phys. Rev. Lett. 49, 460 (1982).

[12] I. V. Khalzov and A. I. Smolyakov, On the calculation of steady-state magnetohydrodynamic flows of liquid metals in circular ducts of a rectangular cross-section, Tech. Phys. 51, 26 (2006).

[13] J. Boisson, R. Monchaux, and S. Aumaître, Inertial regimes in a curved electromagnetically forced flow, J. Fluid Mech. 813, 860 (2017).

[14] R. M. Digilov, Making a fluid rotate: Circular flow of a weakly conducting fluid induced by a Lorentz body force, Am. J. Phys. 75, 361 (2007)

[15] S. A. Suslov, J. Pérez-Barrera, and S. Cuevas, Electromagnetically driven flow of electrolyte in a thin annular layer: axisymmetric solutions, J. Fluid Mech. 828, 573 (2017).

[16] C. Andereck, S. Liu, and H. Swinney, Flow regimes in a circular Couette system with independently rotating cylinders, J. Fluid Mech. 164, 155 (1986).

[17] P. Chossat and G. Iooss, The Couette-Taylor Problem (Springer, New York, 1994).

[18] G. Rüdiger, M. Gellert, R. Hollerbach, M. Schultz, and F. Stefani, Stability and instability of hydromagnetic Taylor-Couette flows, Phys. Rep. 741, 1 (2018).

[19] Y. Zhao and O. Zikanov, Instabilities and turbulence in magnetohydrodynamic flow in a toroidal duct prior to transition in Hartmann layers, J. Fluid Mech. 692, 288 (2012).

[20] E. Sanmiguel-Rojas, J. Ortega-Casanova, C. del Pino, and R. Fernandez-Feria, A Cartesian grid finitedifference method for 2D incompressible viscous flows in irregular geometries, J. Comput. Phys. 204, 302 (2005).

[21] W. H. Press, B. P. Flannery, S. A. Teukolsky, and W. Vettering, Numerical Recipes in C: The Art of Scientific Computing, 2nd ed. (Cambridge University Press, Cambridge, 1992).

[22] W. R. Dean, Fluid motion in a curved channel, Proc. R. Soc. London Ser. A 121, 402 (1928).

[23] A. Pothérat, Three-dimensionality in quasi two-dimensional flows: Recirculations and barrel effects, Eur. Phys. Lett. 98, 64003 (2012).

[24] N. Nivedita, P. Ligrani, and I. Papautsky, Dean flow dynamics in low-aspect ratio spiral microchannels, Sci. Rep. 7, 44072 (2017).

[25] J. A. Baylis, Experiments on laminar flow in curved channels of square section, J. Fluid Mech. 48, 417 (1971).

[26] R. J. Lingwood and T. Alboussière, On the stability of the Hartmann layer, Phys. Fluids 11, 2058 (1999).

[27] D. S. Krasnov, E. Zienicke, O. Zikanov, T. Boeck, and A. Thess, Numerical study of the instability of the Hartmann layer, J. Fluid Mech. 504, 183 (2004). 
[28] A. Thess, D. Krasnov, T. Boeck, E. Zienicke, O. Zikanov, P. Moresco, and T. Alboussière, Transition to turbulence in the Hartmann boundary layer, GAMM-Mitt. 30, 125 (2007).

[29] D. S. Krasnov, O. Zikanov, M. Rossi, and T. Boeck, in Proceedings of 3rd Joint US-European Fluids Engineering Summer Meeting, Montreal, 2010 (ASME, New York, 2010).

[30] D. S. Krasnov, O. Zikanov, M. Rossi, and T. Boeck, Optimal linear growth in magnetohydrodynamic duct flow, J. Fluid Mech. 653, 273 (2010).

[31] Y. Zhao, O. Zikanov, and D. Krasnov, Instability of magnetohydrodynamic flow in an annular channel at high Hartmann number, Phys. Fluids 23, 084103 (2011).

[32] K. Yamamoto, X. Wub, K. Nozakib, and Y. Hayamizu, Visualization of Taylor-Dean flow in a curved duct of square cross-section, Fluid Dyn. Res. 38, 1 (2006)

[33] M. Qin and H. H. Bau, Magnetohydrodynamic flow of a binary electrolyte in a concentric annulus, Phys. Fluids 24, 037101 (2012). 